\title{
Standardization and Accreditation in Haemopoietic Stem Cell Transplantation - an Asia Pacific Perspective
}

\author{
Sharat Damodar ${ }^{1}$, David D Ma ${ }^{2,3}$, Scott J Ragg ${ }^{4}$, Annabella Chang ${ }^{5}$, William YK Hwang ${ }^{6}$, Aloysius YL Ho \\ Alok Srivastava
}

'Dept of Haematology and BMT, Mazumdar shaw medical center, Narayana health city, India, ${ }^{2}$ Department of Haematology and BM Transplant, St Vincent's Hospital Sydney, NSW, Australia, ${ }^{3}$ St Vincent's Clinical School, Faculty of Medicine, UNSW Sydney, NSW, Australia, ${ }^{4}$ Royal Hobart Hospital, Hobart. TAS. Australia, ${ }^{5}$ St Vincent's Centre for Applied Medical Research, Sydney NSW Australia, 'Department of Haematology, Singapore General Hospital, Singapore, 'Dept of Haematology, CMC Vellore, India

\begin{abstract}
Standardization and formal accreditation of practices related to hematopoietic stem cell transplantation (HSCT) and therapies using hematopoietic-derived cellular products aim to promote quality in clinical and laboratory practice and provide knowledge to all stakeholders of centers. This article refers to three aspects of these processes starting with the importance of accurate viable CD 34 enumeration in HSCT. A highly accurate method of enumeration and a robust EQAS program is required, especially during the current COVID-19 pandemic. The second section shares experiences with FACT-JACIE accreditation at the Singapore General Hospital demonstrating how accreditation is part of continuous improvement and not only a destination. This journey can be difficult in many HSCT centers of low- and middle-income countries (LMICS) because of the intensive and rigorous requirements of the internationally accredited models. Hence, in LMICs, a staged movement toward establishing such standards must be considered. This approach is presented in the third section of the article with data on the current situation in countries reporting to the APBMT registry.
\end{abstract}

Key words HSCT, standards, accreditation

Submitted August 17, 2021; Accepted August 25, 2021; Published online October 14, 2021; Issued online October 14, 2021

Correspondence: Sharat Damodar, Department of Haematology and BMT, Mazumdar Shaw medical center, 258/A Bommasandra industrial area, Anekal taluk, Bangalore, India, 560099, E-mail: drsharat.damodar@gmail.com

This article was created from selected presentation at the 26th Annual Congress of APBMT.

\section{Introduction}

The Joint Accreditation Committee, ISCT, and EBMT (JACIE) and Foundation for the Accreditation of Cellular Therapy (FACT) accreditation systems stand out as excellent examples of profession-driven initiatives to improve patient outcomes in hematopoietic stem cell transplantation (HSCT), which have subsequently been incorporated by third parties, such as healthcare payers (health insurers, social security) and competent authorities (treatment authorization) ${ }^{1}$.

CD 34 enumeration is a critical aspect of the HSCT procedure. We understand the importance of standardi- zation and accreditation in the accurate enumeration of these cells. The importance of turnaround time (TAT) and variability in the CD34 numbers between laboratories is another critical aspect that has been highlighted.

The processes and difficulties involved in obtaining FACT-JACIE accreditation have been discussed with the Singapore General Hospital as an example of achieving this accreditation. However, these standards are extremely difficult to incorporate and follow in the lowand middle-income countries (LMICs).

Considerable literature indicating a better clinical outcome in teaching hospitals and centers of excellence has been available since the 1990s. There is evidence of 
a positive relationship between the implementation of a quality management system and the outcome of HSCT, which showed that patient outcomes were systematically better when the transplantation center was at a more advanced phase of JACIE accreditation, independent of the year of transplantation and other risk factors ${ }^{1}$.

Similar studies showed that centers accredited by both FACT and Clinical Trial Network (CTN) demonstrated significantly better results for more complex HSCT, such as HLA-mismatched transplants.

These data reinforce the concept that clinical improvement is driven by the implementation of a quality management system embedded in external accreditation standards, especially in the context of more complex procedures. This process also results in a wider standardization of procedures across different countries and geographic areas, thereby contributing to providing patients with similar treatment expectations even when accessing different health management systems ${ }^{1}$.

\section{Importance of Standardization and Accreditation of CD34+ Stem cell Enumeration Using Flowcy- tometry for Hematopoietic Stem Cell Transplant (HSCT)}

Pioneering studies by Nobel laureate, ED Thomas, and others on HSCT that commenced over six decades ago have established allogeneic and autologous HSCT as a standard of care for patients with life-threatening hematological and non-hematological diseases ${ }^{2}$. In 2015, the Worldwide Network for Blood and Marrow Transplantation reported that, by December 2012, approximately one million HSCT procedures have been performed globally at 1,516 transplant centers across 75 countries $^{3}$. The Asia-Pacific Blood and Marrow Transplantation Group (APBMT) is a contributing member of the Worldwide Network and has registered a similar but significantly rapid rise in the rate of HSCT per year in our region in the last two decades. The increase in international co-operations, such as procurement of unrelated donor products across the globe, exchange of information, and participation in multi-center clinical studies, mean that standardization and accreditation of transplant procedures, including enumeration of hematopoietic stem cells (HSCs), are essential for maintaining and improving patient outcomes.

For decades, bone marrow $(\mathrm{BM})^{4}$ has been the only means of harvesting hematopoietic stem cells for transplantation, and BM nucleated cell enumeration was used to assess the adequacy of BM products for hematopoietic engraftment post-transplant. BM nucleated cell enumeration is an inaccurate measurement of the number of HSCs, as this method measures large numbers of myeloid and lymphoid cells in various stages of matu- ration in addition to HSCs. In contrast, the colony formation unit (CFU) assay, a cell culture-based functional assay for $\mathrm{HSCs}^{4}$, remains a useful test for evaluating post manipulations of HSCT products, such as cryopreservation, $\mathrm{T}$ cell depletion, and major $\mathrm{ABO}$ blood group mismatched transplants. The major drawbacks of the CFU assay are the slow TAT of 14 days and a labor-intensive bioassay that is difficult to standardize.

The discovery of the ability to harvest HSCs from the peripheral blood (PB) after chemotherapy and GCSF was reported in the late 1980 s by our colleagues in Adelaide, Australia ${ }^{5}$, which accelerated the need for a rapid quantitative test for HSCs to suit the collection of HSCs from the blood by apheresis. The enumeration of total CD34+ HSCs using flow cytometry with same day TAT was demonstrated to correlate with the success of hematopoietic engraftment post-transplant and a suitable test to guide PBSC harvest ${ }^{6}$. However, published studies, including ours in Australasia ${ }^{7}$, demonstrated clinically unacceptable variations in CD34+ cell enumeration among laboratories. This led to the establishment of guidelines for the standardization of CD34+ cell enumeration $^{8}$ and external quality assurance (EQA) programs among HSCT facilities. Moreover, studies by our team in Sydney ${ }^{7,9}$ led to the establishment of a CD34+ cell EQA program by the Royal College of Pathologists of Australasia in the early 2000s. This EQA program is used by over 60 HSCT laboratories located predominantly in the Asia-Pacific region.

The 2021 edition of the FACT-JACIE International Standards for Hematopoietic Cellular Therapy Product Collection, Processing, and Administration requires an assay measuring viable CD34 (vCD34+) be performed for HPC products intended for restoration of hematopoiesis ${ }^{10}$. CD34 is a cell surface protein used to quantify the total number of CD34+ cells in a sample, and viability exclusion dyes, such as 7-AAD, are needed to assess the viability of these cells ${ }^{11}$. As fixed and stabilized cells are used for total CD34+ EQA programs, they are unsuitable for cell viability testing. A multicentered study published in $2020^{12}$ has raised concerns on the highly variable vCD34+ cell recovery between collection and transplant facilities on cryopreserved SC products obtained during COVID-19 global travel restrictions and suggested that the lack of standardization of vCD34+ cell assay may be a major contributing factor. This highlights the urgency to establish EQA programs for standardization and accreditation of the $\mathrm{vCD}$ 34+ assay. Attempts to harmonize the enumeration of vCD34+ cells in frozen cord blood products have been performed recently ${ }^{13}$, as the infusion dose of allogeneic cord blood products correlates with engraftment ${ }^{14}$. To date, there have been no reports of an established EQA program for vCD34+ cell counts. The obstacles of es- 
tablishing a commercially workable and affordable vCD 34 EQA program include logistics and cost of procurement, storage, and timely dispatch of cryopreserved human HSCs to participating laboratories. Possible solutions to these challenges are being investigated by various groups, including our team (personal communication), and the urgency has been accelerated by the current pandemic, as the transportation of fresh HSC products among national and international HSCT facilities has been replaced by cryopreserved products.

\section{FACT-JACIE Accreditation at the Singapore General Hospital}

The Foundation for the Accreditation of Cellular Therapy (FACT) and the Joint Accreditation Committee (ISCT) and EBMT (JACIE) have collaborated since 2002 to publish the FACT-JACIE International Standards for Hematopoietic Cellular Therapy Product Collection, Processing, and Administration. The standards, which are in their seventh edition, aim to promote quality medical and laboratory practice in hematopoietic progenitor cell transplantation and related therapies using hematopoietic derived cellular products. These standards apply to hematopoietic progenitor cells, nucleated cells, mononuclear cells, and immune effector cells. Both FACT and JACIE accredit centers based on a review of submitted documents and an on-site inspection for compliance with the current edition of FACT-JACIE Standards ${ }^{11}$. Accreditation will cover the clinical program and, if applied for, will include cell collection and cell processing facilities. Accreditation for immune effector cell therapy may be applied either jointly or separately. AABB, in applying its core principles of quality and donor and patient for blood banks, is another organization that accredits cellular therapy and is accredited by the International Society for Quality in Healthcare for its high standards ${ }^{15}$.

Accreditation of hematopoietic stem cell transplant centers helps inform patients, insurance organizations, and governments of the high standards of these centers. Accredited centers have better adherence to standards and better survival outcomes ${ }^{16}$. However, other factors, such as participation in clinical trial networks, also play a role ${ }^{17}$.

As part of ongoing efforts to continuously improve cellular therapy, the Singapore General Hospital hematopoietic stem cell transplant program obtained AABB accreditation in 2012 and FACT-JACIE accreditation in 2016. Common aspects of these accreditation programs include the following:

1. Standard operating procedures for all critical processes in the organization.

2. Validation that the above SOPs can deliver the de- sired outcomes.

3. Validated and documented training programs for staff for all critical processes.

4. Processes for deliberate procedural deviations.

5. Processes for corrective and preventive action.

6. Document control to ensure that only the latest validated SOPs and forms are used.

7. Record control to ensure that all critical data are properly recorded with strict processes to control any necessary corrections.

8. Benchmarking, which is now integrated into the FACT-JACIE standards, is available for EBMT members.

Staff involved in the process of accreditation include the following:

1. Hematopoietic stem cell transplant physicians, hematologists, and oncologists involved in the care of general patients. Involvement of intensive care unit physicians and infectious disease specialists is also helpful.

2. Transplant coordinators involved in coordinating the entire transplant process, including patient suitability assessment, transplant scheduling, and posttransplant follow-up. They may be nursing or allied health staff and involved in donor management, including HLA typing, donor selection, donor assessment harvesting of stem cells, and coordination of cell transport and shipment.

3. Nursing staff - inpatient and outpatient.

4. Pheresis staff who help ensure safe, efficient, and high-quality leukapheresis of mobilized stem cells.

5. Laboratory staff involved in handling, testing, and processing of cells.

6. Operating theatre staff for bone marrow harvesting.

7. Blood bank staff involved in testing and handling of blood products.

8. Hospital administrative leadership and staff to garner support from multidisciplinary teams and provide necessary administrative support.

The process of accreditation should be managed like any change in management program. Applying Kotter's eight-step change management process $^{18}$, the useful steps include the following:

1. Creating a sense of urgency - helping others see the need for change and communicating the importance of immediate action.

2. Build a guiding coalition - a group of people who are effective and motivated to guide, coordinate, and communicate the plan.

3. Form a strategic vision and initiative - by clarifying how the future will be different from the past and how that future can be a reality through initiatives linked directly to the vision.

4. Enlist a "volunteer army" - accreditation is not 
Table 1. Survey of HSCT accreditation/standardization of practices in APBMT region

\begin{tabular}{rlrrlr}
\hline S. No & $\begin{array}{c}\text { Country/ } \\
\text { Region }\end{array}$ & $\begin{array}{c}\text { No. of } \\
\text { HSCT } \\
\text { centers }\end{array}$ & S. No & $\begin{array}{c}\text { Country/ } \\
\text { Region }\end{array}$ & $\begin{array}{c}\text { No. of } \\
\text { HSCT } \\
\text { centers }\end{array}$ \\
\hline 1 & Bangladesh & 4 & 11 & Sri Lanka & 4 \\
\hline 2 & China & 169 & 12 & Nepal & 1 \\
\hline 3 & Hong Kong & 8 & 13 & Japan & 345 \\
\hline 4 & Indonesia & 3 & 14 & Thailand & 12 \\
\hline 5 & India & 105 & 15 & Australia & 42 \\
\hline 6 & Iran & 18 & 16 & Philippines & 5 \\
\hline 7 & Taiwan & 17 & 17 & Vietnam & 2 \\
\hline 8 & Myanmar & 2 & 18 & Singapore & 4 \\
\hline 9 & Malaysia & 16 & 19 & New Zealand & 6 \\
\hline 10 & Korea & 44 & 20 & Pakistan & 9 \\
\hline
\end{tabular}

a one-person job nor a small team effort. The preceding list of staff is part of those who will need to be fully convinced and subscribed to this effort.

5. Enable action by removing barriers - inefficient processes, silos, and hierarchies are barriers to improvement. Staff should be encouraged to speak up and work collectively toward improvement.

6. Generate short-term wins - intermediate levels of accreditation, including hospital licensing and national accreditation, could be helpful here.

7. Sustain acceleration - by pressing harder after the initial successes.

8. Institute change - by remembering accreditation is not only a goal, but a means of ensuring high-quality standards.

Finally, it is important to remember that accreditation is part of a journey of continuous improvement and not only a destination. While it is a lofty goal, the process of working towards it leads to higher standards in any organization.

\section{Hsct Standards in Low- and Middle-income Countries}

Approximately 20 years ago, the complexity of HSCT and its complications led to efforts by transplantation professionals to standardize processes based on consensus as a way to better manage the inherent risks of this treatment. HSCT has been a pioneer in the area of quality and standards ${ }^{11}$.

In 1998, EBMT and the International Society for Cell and Gene Therapy (ISCT) established the Joint Accreditation Committee, ISCT, and EBMT (JACIE), aiming to offer an inspection-based accreditation process in HSCT against established international standards. JACIE is a committee of the EBMT, whose members are appointed by and are accountable to the EBMT
Table 2. Quality programs for HSCT in APBMT region

\begin{tabular}{lrr}
\hline & Yes (\%) & No (\%) \\
\hline $\begin{array}{l}\text { Are there defined standards for HSCT cen- } \\
\text { ters in your country/region }\end{array}$ & $14(70)$ & $6(30)$ \\
\hline $\begin{array}{l}\text { Are these defined standards mandatory } \\
\text { Are the defined standards as per international } \\
\text { norms (FACT/JACIE) }\end{array}$ & $7(50)$ & $7(50)$ \\
$\begin{array}{l}\text { Are these defined standards as per country/ } \\
\text { region specific norms }\end{array}$ & $9(64)$ & $5(36)$ \\
$\begin{array}{l}\text { Are these defined standards as per institu- } \\
\text { tional/HSCT center defined norms }\end{array}$ & $6(43)$ & $8(57)$ \\
$\begin{array}{l}\text { Is there a system of audit of HSCT centers } \\
\text { for maitenance of standards }\end{array}$ & $5(36)$ & $9(64)$ \\
\hline
\end{tabular}

Board, and ISCT is represented by two members of the Committee. JACIE collaborates with the US-based Foundation for the Accreditation of Cellular Therapy (FACT) to develop and maintain global standards for the provision of quality medical and laboratory practice in cellular therapy ${ }^{11}$.

There have been studies on the improvement of clinical outcomes of HSCT, in which standards and accreditation policies have been put into practice. Initial evidence of a positive relationship between the implementation of a quality management system and the outcome of HSCT in Europe was published in $2011^{19}$, where patient outcomes were systematically better when the transplantation center was at a more advanced phase of the JACIE accreditation, independent of the year of transplantation and other risk factors. Based on another analysis performed on a large cohort of patients who received either allogeneic or autologous transplantation between 1996 and 2006, the decrease in overall mortality in allogeneic procedures over the 14-year observation period was significantly faster in JACIE-accredited centers, thus resulting in higher relapse-free survival and overall survival at 72 months after transplantation ${ }^{20}$. These data reinforce the need to define standard procedures and practices in stem cell transplantation across centers, countries, and regions to provide uniform clinical care. A comprehensive review of this topic has been recently published ${ }^{21}$.

The seventh edition of the JACIE standards has 995 line items. Following these standards in the LMIC regions has been almost impossible for more than $95 \%$ of the centers. Hence, from a practical perspective, these were divided into three levels, one, two, and three, with the aim of providing a graded approach to try and achieve these standards over a period of time. However, we realized that $65 \%$ of the items fell into level 1 , which was also difficult for most centers in the LMIC region. Hence, level 1 was further subdivided into $1 \mathrm{~A}$ and $1 \mathrm{~B}$ to further ease the process of fulfilling these requirements. The idea was to establish a level of mini- 
mum standards for each country, which could be easily followed and thereby help in the development with an ultimate goal of achieving international standards. This more graded approach is possible with most centers in the Asia-Pacific region. Depending on the applicability of this approach, more country-specific approaches may be needed to formalize maintaining the principles of quality management.

While establishing an HSCT program in a developing country, certain common challenges must be considered, such as financial, technological, logistic, social, and availability of skilled manpower. Given the exponential growth in the number of HSCTs worldwide and the establishment of new HSCT centers in high- and low-income countries, the Worldwide Network for Blood and Marrow Transplantation (WBMT) has recognized the need to provide guidance to institutions and individuals who are considering the start of a new HSCT center. Part I of this review describes the absolute minimum, minimum, preferred, and ideal requirements in establishing a new HSCT program. Part II describes the clinical, technical, and financial considerations for establishing an HSCT program in a resourceconstrained setting (typical for developing countries) ${ }^{22}$.

In many healthcare settings, benchmarking for complex procedures has become a mandatory requirement by competent authorities, regulators, payers, and patients to ensure clinical performance, cost-effectiveness, and safe care of patients. In several countries inside and outside Europe, benchmarking systems have been established for HSCT, although access is not universal. As benchmarking is now integrated into the FACT-JACIE standards, the EBMT and JACIE established a Clinical Outcomes Group (COG) to develop and introduce a universal system accessible across EBMT members ${ }^{23}$. This system of benchmarking for HSCT can be established in the APBMT region.

While performance since the introduction of the JACIE quality management system has improved for allogeneic HSCT, the impact on autologous HSCT remains unclear in Europe. A study from 17 Belgian centers showed a relatively homogeneous performance between Belgian centers before the national completeness of JACIE implementation. The three center-related factors had a significant impact on 1-year survival, while activity volume and type of HSCT impacted the 3-year survival of autologous-HSCT patients in univariable analyses. Only the activity volume (impact on 1-year survival only) and type of HSCT (impact on 1- and 3year survivals) remained significant in the multivariable analysis $^{24}$.

We conducted a survey of HSCT centers in the APBMT region to examine the current practices of implementing HSCT standards in the respective countries
(Table 1 and 2). In total, 70\% of countries had some standards defined, of which $70 \%$ were not as per FACT/JACIE. Moreover, $70 \%$ of countries followed these local standards, even though it was mandatory in only $50 \%$. In addition, $65 \%$ of countries did not have a system to audit these standards.

\section{Conclusion}

Standards and accreditations are a vital part of a successful stem cell transplant unit. There is clear evidence that centers that are accredited or in the process of accreditation have better patient outcomes. All processes need to be standardized with robust EQAS programs for the entire region and harmonized with global standards. The accreditation process can be accomplished in a graded manner, especially for centers in the LMICs, in which a step-wise approach needs to be defined and laid out. We believe that adherence to defined standards is essential in improving HSCT practices, which can be locally defined but well calibrated with international norms. Establishing these standards improves outcomes and adds to the volume and types of transplants performed at the centers.

\section{Author Contributions}

Authors S.A and A.S. wrote the introduction, conclusions and section on LMIC, Authors DDM, SJR and $\mathrm{AC}$ write the section on standardization of CD34, WYKH and AYH wrote the section on FACT-JACIE in $\mathrm{SGH}$. All authors reviewed, revised and approved final version of the manuscript.

\section{Conflict of interest}

The authors declare no conflicts of interest associated with this article. Disclosure forms provided by the authors are available on the website.

Alok Srivastava is one of the Editors of Blood Cell Therapy. He was not involved in the editorial evaluation or decision to accept this article for publication.

\section{References}

1. Saccardi R, McGrath E, Snowden JA. JACIE Accreditation of HSCT Programs. The EBMT Handbook: Hematopoietic Stem Cell Transplantation and Cellular Therapies. 7th edition.

2. Appelbaum FR. Hematopoietic-cell transplantation at 50. N Engl J Med. 2007; 357: 1472-5.

3. Gratwohl A, Pasquini MC, Aljurf M, Atsuta Y, Baldomero H, Foeken L, et al. One million haemopoietic stem-cell transplants: a retrospective observational study. Lancet Haematol. 2015; 2: e91-100.

4. Ma D. Hematopoietic reconstitution following bone marrow 
transplantation.: Raven Press Ltd.; 1990.

5. To LB, Shepperd KM, Haylock DN, Dyson PG, Charles P, Thorp DL, et al. Single high doses of cyclophosphamide enable the collection of high numbers of hemopoietic stem cells from the peripheral blood. Exp Hematol. 1990; 18: 442-7.

6. Siena S, Schiavo R, Pedrazzoli P, Carlo-Stella C. Therapeutic relevance of $\mathrm{CD} 34$ cell dose in blood cell transplantation for cancer therapy. J Clin Oncol. 2000; 18: 1360-77.

7. Chang A, Ma DD. The influence of flow cytometric gating strategy on the standardization of CD34+ cell quantitation: an Australian multicenter study. Australasian BMT Scientists Study Group. J Hematother. 1996; 5: 605-16.

8. Sutherland DR, Anderson L, Keeney M, Nayar R, Chin-Yee I. The ISHAGE guidelines for CD34+ cell determination by flow cytometry. International Society of Hematotherapy and Graft Engineering. J Hematother. 1996; 5: 213-26.

9. Chang A, Raik E, Marsden K, Ma DD. Australasian CD34+ quality assurance program and rationale for the clinical utility of the single-platform method for CD34+ cell enumeration. Cytotherapy. 2004; 6: 50-61.

10. EBMT. TFftAoCTatJACIa. FACT-JACIE International Standards for Hematopoietic Cellular Therapy Product Collection, Processing, and Administration Eighth Edition ed; 2021.

11. Keeney M, Chin-Yee I, Weir K, Popma J, Nayar R, Sutherland DR. Single platform flow cytometric absolute CD34+ cell counts based on the ISHAGE guidelines. International Society of Hematotherapy and Graft Engineering. Cytometry. 1998; 34: 61-70.

12. Purtill D, Antonenas V, Chiappini P, Tong D, O'Flaherty E, Bajel A, et al. Variable CD34+ recovery of cryopreserved allogeneic HPC products: transplant implications during the COVID-19 pandemic. Blood Adv. 2020; 4: 4147-50.

13. Fournier D, Lewin A, Simard C, Trepanier P, Neron S, Ballerini L, et al. Multi-laboratory assay for harmonization of enumeration of viable CD34+ and CD45+ cells in frozen cord blood units. Cytotherapy. 2020; 22: 44-51.

14. Avery S, Shi W, Lubin M, Gonzales AM, Heller G, CastroMalaspina $\mathrm{H}$, et al. Influence of infused cell dose and HLA match on engraftment after double-unit cord blood allografts. Blood. 2011; 117: 3277-85; quiz 478.

15. AABB. ABOUT AABB STANDARDS AND ACCREDITATION FOR CELLULAR THERAPIES. 2021.

16. Anthias C, O'Donnell PV, Kiefer DM, Yared J, Norkin M, Anderlini P, et al. European Group for Blood and Marrow Transplantation Centers with FACT-JACIE Accreditation Have Significantly Better Compliance with Related Donor Care Standards. Biol Blood Marrow Transplant. 2016; 22: 514-9.
17. Marmor S, Begun JW, Abraham J, Virnig BA. The impact of center accreditation on hematopoietic cell transplantation (HCT). Bone Marrow Transplant. 2015; 50: 87-94.

18. https://www.kotterinc.com/8-steps-process-for-leading-change

19. Gratwohl A, Brand R, Niederwieser D, Baldomero H, Chabannon C, Cornelissen J, et al. Introduction of a quality management system and outcome after hematopoietic stemcell transplantation. J Clin Oncol. 2011; 29: 1980-6.

20. Gratwohl A, Brand R, McGrath E, van Biezen A, Sureda A, Ljungman $\mathrm{P}$, et al. Use of the quality management system "JACIE" and outcome after hematopoietic stem cell transplantation. Haematologica. 2014; 99: 908-15.

21. Snowden JA, McGrath E, Duarte RF, Saccardi R, Orchard K, Worel N, et al. JACIE accreditation for blood and marrow transplantation: past, present and future directions of an international model for healthcare quality improvement. Bone Marrow Transplant. 2017; 52: 1367-71.

22. Aljurf M, Weisdorf D, Hashmi SK, Nassar A, Gluckman E, Mohty M, et al. Worldwide Network for Blood and Marrow Transplantation (WBMT) recommendations for establishing a hematopoietic stem cell transplantation program in countries with limited resources (Part II): Clinical, technical and socioeconomic considerations. Hematol Oncol Stem Cell Ther. 2020; 13: 7-16.

23. Snowden JA, Saccardi R, Orchard K, Ljungman P, Duarte RF, Labopin $\mathrm{M}$, et al. Benchmarking of survival outcomes following haematopoietic stem cell transplantation: A review of existing processes and the introduction of an international system from the European Society for Blood and Marrow Transplantation (EBMT) and the Joint Accreditation Committee of ISCT and EBMT (JACIE). Bone Marrow Transplant 2020; 55: 681-94. doi: 10.1038/s41409-019-0718-7.

24. Poirel HA, Vanspauwen M, Macq G, De Geyndt A, Maertens J, Willems E, et al. Providing both autologous and allogeneic hematopoietic stem cell transplants (HSCT) may have a stronger impact on the outcome of autologous HSCT in adult patients than activity levels or implementation of JACIE at Belgian transplant centres. Bone Marrow Transplant 2019; 54: $1434-42$.

https://doi.org/10.31547/bct-2021-015

Copyright (C)2021 Asia-Pacific Blood and Marrow Transplantation Group (APBMT). This is an open access article distributed under CC BY-NC license (https://creativecommon s.org/licenses/by-nc/4.0/). 